\title{
FREE CONVECTION HEAT AND MASS TRANSFER OF A NANOFLUID PAST A HORIZONTAL CYLINDER EMBEDDED IN A NON-DARCY POROUS MEDIUM
}

\author{
A. Subba Rao, ${ }^{1, *}$ V.R. Prasad, ${ }^{1}$ O. Anwar Bég, ${ }^{2}$ \& M. Rashidi ${ }^{3}$ \\ ${ }^{1}$ Department of Mathematics, Madanapalle Institute of Technology and Science, Madanapalle, \\ India \\ ${ }^{2}$ Fluid Mechanics, Nanosystems and Propulsion, Aeronautical and Mechanical Engineering, \\ School of Computing, Science and Engineering, Newton Building, University of Salford, \\ Manchester M54WT, United Kingdom \\ ${ }^{3}$ Mechanical Engineering Department, Engineering Faculty of Bu-Ali Sina University, \\ Hamedan, Iran
}

*Address all correspondence to: A. Subba Rao, Department of Mathematics, Madanapalle Institute of Tech-
nology and Science, Madanapalle, India, E-mail: asrsvu@gmail.com

Original Manuscript Submitted: 6/25/2015; Final Draft Received: 2/15/2017

\begin{abstract}
In the present paper, we analyzed the laminar boundary layer flow and heat transfer from a horizontal cylinder in a nanofluid-saturated non-Darcy porous medium in the presence of thermal radiation. This is the first paper presenting non-similar solutions for such a regime. The boundary layer conservation equations, which are parabolic in nature, are normalized into non-similar form and then solved computationally with an efficient, implicit, stable Keller-box finitedifference scheme. Non-Darcy effects are simulated via a second-order Forchheimer drag force term in the momentum boundary layer equation. The model used for the nanofluid incorporates the effects of Brownian motion, buoyancy ratio, and thermophoresis. A non-similarity solution is presented that depends on the Brownian motion number $(\mathrm{Nb})$, buoyancy ratio $(\mathrm{Nr})$, thermophoresis number $(\mathrm{Nt})$, Forchheimer parameter $(\Lambda)$, and radiation parameter $(F)$. Velocity is reduced with increasing Forchheimer parameter, whereas temperature and nanoparticle concentration are both enhanced. The model finds applications in energy systems and thermal enhancement of industrial flow processes.
\end{abstract}

KEY WORDS: Keller-box method, nanofluids, thermal radiation, horizontal cylinder, Brownian motion, Forchheimer number

\section{INTRODUCTION}

Transport processes in porous media can involve fluid, heat, and mass transfer in single or multi-phase scenarios. Such flows with and without buoyancy effects arise frequently in many branches of chemical engineering, and owing to their viscous-dominated nature are generally simulated using the Darcy model. Applications of such flows include chip-based microfluidic chromatographic separation devices (Dorfman and Brenner, 2002). Porous media flow simulations are also critical in convective processes in hygroscopic materials (Turner et al., 1998), such as electroremediation in the soil decontamination technique wherein an electric field applied to a porous medium generates the migration of ionic species in solution (Pomès et al., 2002). Both Darcian and Darcy-Forchheimer (inertial) models have been employed extensively in radiative-convective flows in porous media. Takhar et al. (1998) used an implicit difference scheme and the Cogley-Vincenti-Giles non-gray model to simulate the radiation-convection gas flow in a non-Darcy porous medium with viscous heating effects. Nagaraju et al. (2001) used the Schuster-Schwartzchild two-flux radiative model and the Blottner finite-difference scheme to investigate the combined radiative and convective heat transfer in a medium with variable porosity. Takhar et al. (2003) employed a Runge-Kutta-Merson 
shooting quadrature and the Rosseland diffusion algebraic radiation model to analyze the mixed radiation-convection flow in a non-Darcy porous medium, showing that temperature gradients are boosted with radiative flux. More recently, Chamkha et al. (2004) studied the influence of thermal radiation on steady natural convection in a viscoelastic fluid-saturated non-Darcian porous medium using the Keller-box numerical scheme. Temperatures were seen to be substantially boosted with an increase in radiative parameter. Hossain and Pop (2001) studied radiation effects on free convection over a flat plate embedded in a porous medium with high porosity. Takhar et al. (2002) reported natural convection on a vertical cylinder embedded in a thermally stratified high-porosity medium.

Nanofluids are engineered colloids comprising a base fluid (e.g., air and water) and nanoparticles. Nanoparticles range in diameter between 1 and $100 \mathrm{~nm}$. Nanofluids typically employ metal or metal oxide nanoparticles, such as copper and alumina, and the base fluid is usually a conductive fluid, such as water or ethylene glycol. Nanofluids commonly contain up to a 5\% volume fraction of nanoparticles to ensure effective heat transfer enhancements. Nanofluids are studied due to their heat transfer properties: they enhance the thermal conductivity and convective properties over the properties of the base fluids. Typical thermal conductivity enhancements are in the range of $15 \%-$ $40 \%$ over the base fluid and heat transfer coefficient enhancements have been found up to $40 \%$ (Yu et al., 2008). Increases in thermal conductivity of this magnitude cannot be solely attributed to the higher thermal conductivity of the added nanoparticles, and other mechanisms attributable to the increase in performance. Fluid heating and cooling techniques are important in many industries such as power, manufacturing, transportation, and in particular the next generation of thin film solar energy collector devices. Effective cooling techniques are greatly needed for cooling any sort of high-energy device. Common heat transfer fluids such as water, ethylene glycol, and engine oil have limited heat transfer capabilities due to their low heat transfer properties. In contrast, metals have thermal conductivities up to three times higher than these fluids; therefore, it is naturally desirable to combine the two substances to produce a heat transfer medium that behaves like a fluid but has the thermal conductivity of a metal. Innovative heat transfer fluids containing suspended nanometer-sized solid particles (i.e., nanofluids) can therefore beneficially change the transport and thermal properties of the base fluid and offer great promise for modern heat transfer engineering. A comprehensive survey of convective transport in nanofluids was made by Buongiorno (2006), who considered seven slip mechanisms that can produce a relative velocity between the nanoparticles and the base fluid: inertia, Brownian diffusion, thermophoresis, diffusiophoresis, Magnus effect, fluid drainage, and gravity. Of all of these mechanisms, only Brownian diffusion and thermophoresis were found to be important. In the analysis, Buongiorno (2006) used a two-component equilibrium model for mass, momentum, and heat transport in nanofluids, and showed that energy transfer by nanoparticle dispersion is negligible and cannot explain the abnormal heat transfer coefficient increases. An excellent assessment of nanofluid physics and developments has been provided by Das et al. (2007). Buongiorno and $\mathrm{Hu}$ (2005) observed that although convective heat transfer enhancement has been suggested to be due to the dispersion of suspended nanoparticles, this effect however is too small to explain the observed enhancement. They further assert that turbulence is not affected by the presence of nanoparticles, thus this cannot explain the observed enhancement.

The aforementioned studies did not consider transport in porous media. Such flows are very important, for example, in fuel cell technologies, geothermics, materials processing, trickle bed chromatography, etc. Coupled heat and mass transfer in free convection boundary layer flows in porous media arise in many such applications. A large proportion of studies utilize the Darcy model, which is valid for low Reynolds number flows (Bear, 1988). Important studies in this regard have been made by Bejan and Khair (1985), Lai and Kulacki (1991), and Murthy and Singh (1999). Further analyses include stretching sheet hydromagnetic flow in porous media (Bég et al., 2009), ReinerRivlin differential fluid transport in a porous regime (Bég et al., 2008), viscoelastic convection from a wedge in Darcy-Brinkman porous media (Bég et al., 2004), chemically reactive thermo-micropolar transport in porous media (Bég et al., 2007), and two-phase magneto-convection in porous media (Zueco and Bég, 2009). Other recent studies employing non-Darcy transport models have been conducted within the context of chemical process and medical engineering (Bég et al., 2012), nanofluids and porous medium (Xiao et al., 2013; Cai and Yu, 2011; Rashidi et al., 2014a,b,c), and radiation (Rashidi et al., 2014d).

To the authors' knowledge no studies have thus far been communicated with regard to nanofluid thermal convection from a cylinder in non-Darcian porous media. The objective of the present paper is therefore to analyze the development of steady boundary layer flow and heat transfer in nanofluid-saturated, isotropic, homogenous porous 
media and radiation, for the case of a horizontal cylinder. A non-similarity solution is developed. The Keller-box difference scheme is used to solve the normalized boundary layer equations and the effects of the Forchheimer parameter $(\Lambda)$, Brownian motion number $(\mathrm{Nb})$, thermophoresis number $(\mathrm{Nt})$, buoyancy ratio parameter $(\mathrm{Nr})$, and thermal radiation parameter $(F)$ on the relevant flow variables are described in detail. The present study is of immediate interest in next-generation solar film collectors, heat exchanger technology, and also geothermal energy storage systems using spherical subterranean tanks. The present problem has to the authors' knowledge not appeared thus far in the scientific literature and is relevant to polymeric thermal enrobing processes immersed in a porous medium.

\section{MATHEMATICAL MODEL}

Consider the steady, laminar, two-dimensional, incompressible heat and mass transfer flow of a nanofluid past a horizontal permeable cylinder embedded in an isotropic, homogenous, fully saturated porous medium. Unidirectional radiative flux is present. The regime constitutes an enrobing flow scenario. Figure 1 illustrates the flow geometry and physical coordinate system. The $x$-coordinate is measured along the circumference of the horizontal cylinder from the lowest point and the $y$-coordinate is measured normal to the surface, with $a$ denoting the radius of the horizontal cylinder. Here, $\Phi=x / a$ is the angle of the $y$-axis with respect to the vertical $(0 \leq \Phi \leq \pi)$. The gravitational acceleration $g$, acts downward. We also assume that the Boussineq approximation holds, i.e., density variation is only experienced in the buoyancy term in the momentum equation.

Both the horizontal cylinder and the nanofluid are maintained initially at the same temperature. Instantaneously, they are raised to a temperature $T_{w}\left(>T_{\infty}\right)$ and concentration $C_{w}\left(>C_{\infty}\right)$, which remain unchanged. The OberbeckBoussinesq approximation is employed. Homogeneity and local thermal equilibrium in the porous medium is assumed. We consider a porous medium with porosity denoted by $\varepsilon$ and permeability (hydraulic conductivity) denoted by $K$. The following equations embody the conservation of total mass, momentum, thermal energy, and nanoparticles, respectively. The field variables are velocity $v$, temperature $T$, and nanoparticle volume fraction $C$ :

$$
\begin{gathered}
\nabla \cdot \mathbf{v}=0 \\
\frac{\rho_{f}}{\varepsilon} \frac{\partial \mathbf{v}}{\partial t}=-\nabla p-\frac{\mu}{K} \mathbf{v}-\Gamma \mathbf{v}^{2}+\left[C \rho_{p}+(1-C)\left\{\rho_{f}\left[1-\beta\left(T-T_{\infty}\right)\right]\right\} \sin (x / a)\right] g \\
(\rho c)_{m} \frac{\partial T}{\partial t}+(\rho c)_{f} \mathbf{v} \cdot \nabla T=k_{m}\left[\nabla^{2} T+\frac{\partial q_{r}}{\partial y}\right]+\varepsilon(\rho c)_{p}\left[D_{B} \nabla C \cdot \nabla T+\left(D_{T} / T_{\infty}\right) \nabla T \cdot \nabla T\right] \\
\frac{\partial C}{\partial t}+\frac{1}{\varepsilon} \mathbf{v} \cdot \nabla C=D_{B} \nabla^{2} C+\left(D_{T} / T_{\infty}\right) \nabla^{2} T
\end{gathered}
$$

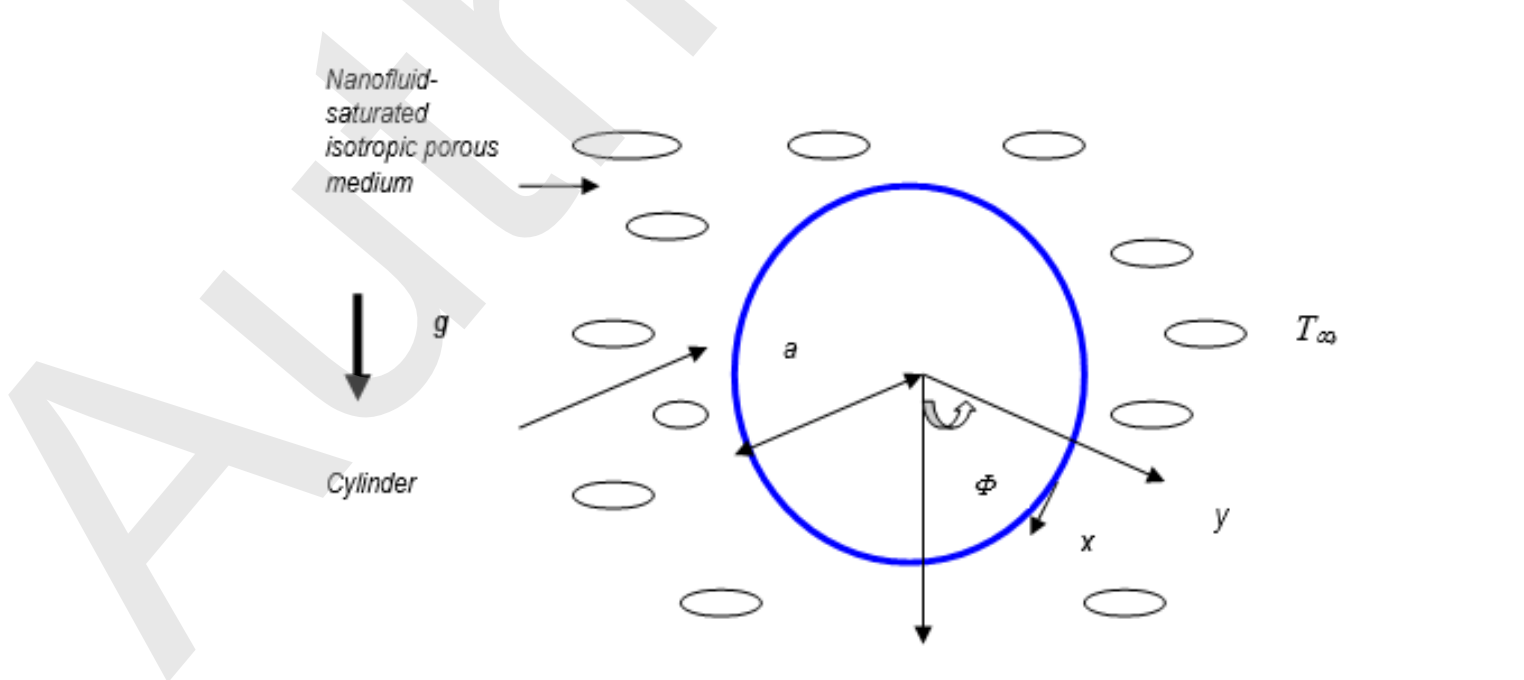

FIG. 1: Physical model and coordinate system 
where $\mathbf{v}=(u, v) ; \rho_{f}, \mu$, and $\beta$ are the density, viscosity, and volumetric volume expansion coefficient of the fluid, respectively; $\rho_{p}$ is the density of the particles; and $\Gamma$ is the Forchheimer inertial coefficient representing secondorder (quadratic) inertial drag effects in the porous medium. The gravitational acceleration is denoted by $g$. We have introduced the effective heat capacity $(\rho c)_{m}$, and the effective thermal conductivity $k_{m}$ of the porous medium. The coefficients that appear in Eqs. (3) and (4) are Brownian diffusion coefficient $D_{B}$ and thermophoretic diffusion coefficient $D_{T}$. Details of the derivation of the Eqs. (3) and (4) are given in Buongiorno (2006), Tzou (2008), and Nield and Kuznetsov (2009).

The boundary conditions are taken to be

$$
\begin{aligned}
& u=v=0, \quad T=T_{w}, \quad C=C_{w} \quad \text { at } \quad y=0 \\
& u=0, \quad T \rightarrow T_{\infty}, \quad C \rightarrow C_{\infty} \quad \text { as } \quad y \rightarrow \infty
\end{aligned}
$$

We consider a steady-state flow. In keeping with the Oberbeck-Boussinesq approximation and an assumption that the nanoparticle concentration is dilute, and with a suitable choice for the reference pressure, we can linearize the momentum equation and write Eq. (2) as

$$
0=-\nabla p-\frac{\mu}{K} \mathbf{v}-\Gamma \mathbf{v}^{2}+\left[\left(\rho_{p}-\rho_{f \infty}\right)\left(C-C_{\infty}\right) \sin (x / a)+\left(1-C_{\infty}\right) \rho_{f \infty} \beta\left(T-T_{\infty}\right) \sin (x / a)\right] g
$$

We now make the standard boundary layer approximation, based on a scale analysis, with extra body force terms incorporated for Darcy impedance and Forchheimer drag (for the porous medium) and this leads to the following equations:

$$
\begin{gathered}
\frac{\partial u}{\partial x}+\frac{\partial v}{\partial y}=0 \\
u \frac{\partial u}{\partial x}+v \frac{\partial u}{\partial y}=\left[\left(1-C_{\infty}\right) \rho_{f \infty} \beta g\left(T-T_{\infty}\right) \sin (x / a)-\left(\rho_{p}-\rho_{f \infty}\right) g\left(C-C_{\infty}\right) \sin (x / a)\right] \\
+v \frac{\partial^{2} u}{\partial y^{2}}-\frac{\mu}{K} u-\Gamma u^{2} \\
u \frac{\partial T}{\partial x}+v \frac{\partial T}{\partial y}=\alpha_{m} \nabla^{2} T+\tau\left[D_{B} \frac{\partial C}{\partial y} \frac{\partial T}{\partial y}+\left(\frac{D_{T}}{T_{\infty}}\right)\left(\frac{\partial T}{\partial y}\right)^{2}\right]-\frac{1}{\rho c_{p}} \frac{\partial q_{r}}{\partial y} \\
\frac{1}{\varepsilon}\left(u \frac{\partial C}{\partial x}+v \frac{\partial C}{\partial y}\right)=D_{B} \frac{\partial^{2} C}{\partial y^{2}}+\left(\frac{D_{T}}{T_{\infty}}\right) \frac{\partial^{2} T}{\partial y^{2}}
\end{gathered}
$$

where

$$
\alpha_{m}=\frac{k_{m}}{(\rho c)_{f}}, \quad \tau=\frac{(\rho c)_{p}}{(\rho c)_{f}}
$$

in which the former is the thermal diffusivity of the nanofluid, and the latter is the ratio between the effective heat capacity of the nanoparticle material and heat capacity of the fluid. Defining the stream function, $\psi$ :

$$
u=\frac{\partial \psi}{\partial y}, \quad v=-\frac{\partial \psi}{\partial x}
$$

the Rosseland diffusion flux model is used and is defined following Modest (1993) as follows:

$$
q_{r}=-\frac{4 \sigma^{*}}{3 k^{*}} \frac{\partial T^{4}}{\partial y}
$$

where $k^{*}$ is the mean absorption coefficient and $\sigma^{*}$ is the Stefan-Boltzmann constant. We further note that the current radiative model is valid for high optical thickness (optical depth), for which thermal radiation is better attenuated in the medium and is known to induce significant heating within boundary layers. Following Raptis and Perdikis (2004), 
we can express the quadratic temperature function in Eq. (14) as a linear function of temperature. The Taylor series for $T^{4}$, discarding higher-order terms, can be shown to give

$$
T^{4} \cong 4 T_{\infty}^{3} T-3 T_{\infty}^{4}
$$

Substituting this expression into Eq. (14) and then into heat conservation Eq. (10), eventually leads to the following form of the energy equation:

$$
u \frac{\partial T}{\partial x}+v \frac{\partial T}{\partial y}=\alpha \frac{\partial^{2} T}{\partial y^{2}}+\tau\left[D_{B} \frac{\partial C}{\partial y} \frac{\partial T}{\partial y}+\left(\frac{D_{T}}{T_{\infty}}\right)\left(\frac{\partial T}{\partial y}\right)^{2}\right]+\frac{16 \sigma^{*} T_{\infty}^{3}}{3 k^{*} \rho c_{p}} \frac{\partial^{2} T}{\partial y^{2}}
$$

Equation (8) is therefore satisfied identically. Proceeding with the analysis we introduce the following dimensionless variables:

$$
\begin{aligned}
\xi & =\frac{x}{a}, \quad \eta=\frac{y}{a} \sqrt[4]{\mathrm{Gr}}, \quad f(\xi, \eta)=\frac{\psi}{v \xi \sqrt[4]{\mathrm{Gr}}} \\
\mathrm{Gr} & =\frac{\left(1-\phi_{\infty}\right) \rho_{f \infty} g \beta\left(T_{w}-T_{\infty}\right) a^{3}}{v^{2}}, \quad \theta(\xi, \eta)=\frac{T-T_{\infty}}{T_{w}-T_{\infty}}, \quad \phi(\xi, \eta)=\frac{C-C_{\infty}}{C_{w}-C_{\infty}}
\end{aligned}
$$

Substituting Eq. (17) into Eqs. (8)-(11), we obtain the coupled, nonlinear, dimensionless partial differential equations for momentum, energy, and species conservation for the regime:

$$
\begin{gathered}
f^{\prime \prime \prime}+f f^{\prime \prime}-(1+\xi \Lambda) f^{\prime 2}+\frac{\sin \xi}{\xi}(\theta-N r \phi)-\frac{1}{\mathrm{Da}} f^{\prime}=\xi\left(f^{\prime} \frac{\partial f^{\prime}}{\partial \xi}-f^{\prime \prime} \frac{\partial f}{\partial \xi}\right) \\
\left(1+\frac{4}{3 F}\right) \frac{\theta^{\prime \prime}}{\operatorname{Pr}}+f \theta^{\prime}+N b \theta^{\prime} \phi^{\prime}+N t\left(\theta^{\prime}\right)^{2}=\xi\left(f^{\prime} \frac{\partial \theta}{\partial \xi}-\theta^{\prime} \frac{\partial f}{\partial \xi}\right) \\
\phi^{\prime \prime}+\operatorname{Sc} f \phi^{\prime}+\left(\frac{N t}{N b}\right) \theta^{\prime \prime}=\xi\left(f^{\prime} \frac{\partial \phi}{\partial \xi}-\phi^{\prime} \frac{\partial f}{\partial \xi}\right)
\end{gathered}
$$

The transformed dimensionless boundary conditions are:

$$
\begin{aligned}
& \text { At } \eta=0: \quad f^{\prime}=0, \quad f=0, \quad \theta=1, \quad \phi=1 \\
& \text { As } \eta \rightarrow \infty: \quad f^{\prime} \rightarrow 0, \quad \theta \rightarrow 0, \quad \phi \rightarrow 0
\end{aligned}
$$

In the previous equations, the primes denote the differentiation with respect to $\eta$, the dimensionless radial coordinate $(\eta)$; $\xi$ is the dimensionless tangential coordinate; $\Phi$ is the azimuthal coordinate; $\Lambda=\Gamma a$ is the local inertia coefficient (the Forchheimer parameter); $\mathrm{Da}=K /\left(\mathrm{Gr}^{1 / 2} a^{2}\right)$ is the Darcy number; $N r=\left[\left(\rho_{p}-\rho_{f \infty}\right)\left(C_{w}-C_{\infty}\right)\right]$ $/\left[\rho_{f \infty}\left(1-C_{\infty}\right) \beta\left(T-T_{\infty}\right)\right]$ is the buoyancy ratio parameter; $\operatorname{Pr}=v / \alpha_{m}$ is the Prandtl number; $\mathrm{Sc}=v / D_{m} \varepsilon$ is the Schmidt number; $N b=\left[\tau D_{B}\left(C_{w}-C_{\infty}\right)\right] / v$ is the Brownian motion parameter; $N t=\left[\tau D_{T}\left(T_{w}-T_{\infty}\right)\right] / v T_{\infty}$ is the thermophoresis parameter; and $F=K k^{*} / 4 \sigma^{*} T_{\infty}^{3}$ is the radiation parameter.

The engineering design quantities of physical interest include the skin-friction coefficient (see Table 1), Nusselt number, and Sherwood number, which are given by

$$
\begin{gathered}
\frac{1}{2} C_{f} \sqrt[4]{\mathrm{Gr}}=\xi f^{\prime \prime}(\xi, 0) \\
\frac{\mathrm{Nu}}{\sqrt[4]{\mathrm{Gr}}}=-\theta^{\prime}(\xi, 0) \\
\frac{\mathrm{Sh}}{\sqrt[4]{\mathrm{Gr}}}=-\phi^{\prime}(\xi, 0)
\end{gathered}
$$


TABLE 1: Values of the local skin-friction coefficient $C_{f}$ for various values of $\xi$ and $\operatorname{Pr}$ with $\mathrm{Nb}=\mathrm{Nt}=\mathrm{Nr}=0$

\begin{tabular}{|c|c|c|c|c|c|c|}
\hline \multirow{2}{*}{$\xi$} & \multicolumn{3}{|c|}{ Pr $=\mathbf{0 . 7 2}$} & \multicolumn{3}{c|}{ Pr $=\mathbf{5 . 0}$} \\
\cline { 2 - 7 } & $\begin{array}{c}\text { Merkin et al. } \\
\mathbf{( 1 9 8 8 )}\end{array}$ & $\begin{array}{c}\text { Prasad et al. } \\
\mathbf{( 2 0 1 4 )}\end{array}$ & $\begin{array}{c}\text { Present } \\
\text { Results }\end{array}$ & $\begin{array}{c}\text { Merkin et al. } \\
\mathbf{( 1 9 8 8 )}\end{array}$ & $\begin{array}{c}\text { Prasad et al. } \\
\mathbf{( 2 0 1 4 )}\end{array}$ & $\begin{array}{c}\text { Present } \\
\text { Results }\end{array}$ \\
\hline 0.0 & 0.000 & 0.000 & 0.000 & 0.000 & 0.000 & 0.000 \\
\hline 0.4 & 0.609 & 0.608 & 0.610 & 0.301 & 0.304 & 0.303 \\
\hline 0.8 & 1.160 & 1.157 & 1.159 & 0.572 & 0.567 & 0.569 \\
\hline 1.2 & 1.602 & 1.598 & 1.600 & 0.787 & 0.784 & 0.784 \\
\hline 1.6 & 1.885 & 1.880 & 1.882 & 0.922 & 0.919 & 0.920 \\
\hline 2.0 & 1.971 & 1.968 & 1.970 & 0.954 & 0.949 & 0.951 \\
\hline 2.4 & 1.824 & 1.820 & 1.821 & 0.865 & 0.861 & 0.863 \\
\hline 2.8 & 1.404 & 1.400 & 1.402 & 0.629 & 0.625 & 0.626 \\
\hline$\pi$ & 0.739 & 0.702 & 0.713 & 0.230 & 0.225 & 0.228 \\
\hline
\end{tabular}

\section{NUMERICAL SOLUTIONS WITH THE KELLER-BOX IMPLICIT METHOD}

The system of Eqs. (18)-(20) subject to boundary conditions (21) have been solved numerically by a very efficient implicit finite-difference scheme called the Keller-box method, which was introduced and succinctly reviewed by Keller (1978). This method remains among the most powerful, versatile, and accurate computational finite-difference schemes employed in modern viscous fluid dynamics simulations. Recent applications in nanofluid dynamics are provided in Yacob et al. (2011) and Tham et al. (2012). Further details are available in Prasad et al. (2014) and Subba Rao et al., (2016a,b). The superior efficiency of this method for parabolic boundary layer flows was also reviewed in Bég et al. (2012) for non-Newtonian magneto-hydrodynamic flows. The method has been validated by numerous published results and allows excellent mesh sensitivity experimentation to optimize the best grid spacing for computing rapidly converging solutions for boundary layers.

In the present study the accuracy of the Keller-box code has been verified by grid independence tests. The key stages are the following:

1. Reduction of the $N$ th order partial differential equation system to $N \times$ first-order equations;

2. Finite-difference discretization;

3. Quasilinearization of the nonlinear Keller algebraic equations; and

4. Block-tridiagonal elimination of the linear Keller algebraic equations.

\subsection{Phase A: Reduction of the Nth Order Partial Differential Equation System to the $N$ First-Order Equations}

Equations (18)-(20) subjected to boundary conditions (21) are first written as a system of first-order equations. For this purpose, we reset Eqs. (18)-(20) as a set of simultaneous equations by introducing the new variables $u, v$, and $t$ :

$$
\begin{aligned}
f^{\prime} & =u \\
f^{\prime \prime} & =v \\
\theta^{\prime} & =t \\
\phi^{\prime} & =p
\end{aligned}
$$




$$
\begin{gathered}
v^{\prime}+f v-(1+\xi \Lambda) u^{2}+\frac{\sin \xi}{\xi}[s-N r(g)]-\frac{1}{\mathrm{Da}} u=\xi\left(u \frac{\partial u}{\partial \xi}-v \frac{\partial f}{\partial \xi}\right) \\
\frac{1}{\operatorname{Pr}}\left(1+\frac{4}{3 F}\right) t^{\prime}+f t+N b(p t)+N t\left(t^{2}\right)=\xi\left(u \frac{\partial s}{\partial \xi}-t \frac{\partial f}{\partial \xi}\right) \\
\frac{1}{\mathrm{Sc}} p^{\prime}+f p+\frac{1}{\mathrm{Sc}}\left(\frac{N t}{N b}\right) t^{\prime}=\xi\left(u \frac{\partial g}{\partial \xi}-p \frac{\partial f}{\partial \xi}\right)
\end{gathered}
$$

where the primes denote differentiation with respect to $\eta$. In terms of the dependent variables, the boundary conditions become

$$
\begin{aligned}
& \text { At } \eta=0: \quad u=0, \quad f=0, \quad s=1, \quad g=1 \\
& \text { As } \eta \rightarrow \infty: \quad u \rightarrow 0, \quad s \rightarrow 0, \quad g \rightarrow 0
\end{aligned}
$$

\subsection{Phase B: Finite-Difference Discretization}

A two-dimensional computational grid is imposed on the $\xi-\eta$ plane as sketched in Fig. 2. The stepping process is defined by

$$
\begin{gathered}
\eta_{0}=0, \quad \eta_{j}=\eta_{j-1}+h_{j}, \quad j=1,2, \ldots, J, \quad \eta_{J} \equiv \eta_{\infty} \\
\xi^{0}=0, \quad \xi^{n}=\xi^{n-1}+k_{n}, \quad n=1,2, \ldots, N
\end{gathered}
$$

where $k_{n}$ and $h_{j}$ denote the step distances in the $\xi$ and $\eta$ directions, respectively. If $g_{j}^{n}$ denotes the value of any variable at $\left(\eta_{j}, \xi^{n}\right)$, then the variables and derivatives of Eqs. (25)-(30) at $\left(\eta_{j-1 / 2}, \xi^{n-1 / 2}\right)$ are replaced by

$$
\begin{gathered}
g_{j-1 / 2}^{n-1 / 2}=\frac{1}{4}\left(g_{j}^{n}+g_{j-1}^{n}+g_{j}^{n-1}+g_{j-1}^{n-1}\right) \\
\left(\frac{\partial g}{\partial \eta}\right)_{j-1 / 2}^{n-1 / 2}=\frac{1}{2 h_{j}}\left(g_{j}^{n}-g_{j-1}^{n}+g_{j}^{n-1}-g_{j-1}^{n-1}\right) \\
\left(\frac{\partial g}{\partial \xi}\right)_{j-1 / 2}^{n-1 / 2}=\frac{1}{2 k^{n}}\left(g_{j}^{n}-g_{j-1}^{n}+g_{j}^{n-1}-g_{j-1}^{n-1}\right)
\end{gathered}
$$

We now state the finite-difference approximation of Eqs. (25)-(30) for the mid-point $\left(\eta_{j-1 / 2}, \xi^{n}\right)$ as follows:

$$
\begin{aligned}
& h_{j}^{-1}\left(f_{j}^{n}-f_{j-1}^{n}\right)=u_{j-1 / 2}^{n} \\
& h_{j}^{-1}\left(u_{j}^{n}-u_{j-1}^{n}\right)=v_{j-1 / 2}^{n}
\end{aligned}
$$

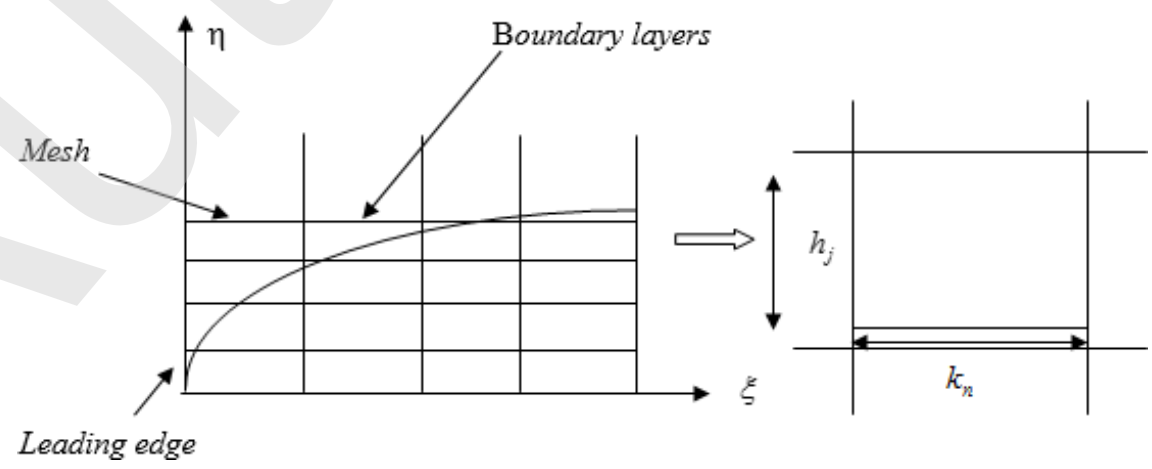

FIG. 2: Grid meshing and a Keller-box computational cell 


$$
\begin{gathered}
\left(\frac{v_{j}^{n}-v_{j-1}^{n}}{h_{j}}\right)+(1+\alpha)\left(f_{j-1 / 2}^{n} v_{j-1 / 2}^{n}\right)-(1+\xi \Lambda+\alpha)\left(u_{j-1 / 2}^{n}\right)^{2}+B\left[s_{j-1 / 2}^{n}-N r\left(g_{j-1 / 2}^{n}\right)\right] \\
-\left(\frac{1}{\mathrm{Da}}\right) u_{j-1 / 2}^{n}+\alpha v_{j-1 / 2}^{n-1} f_{j-1 / 2}^{n}-\alpha f_{j-1 / 2}^{n-1} v_{j-1 / 2}^{n}=\left[R_{1}\right]_{j-1 / 2}^{n-1} \\
h_{j}^{-1}\left(\theta_{j}^{n}-\theta_{j-1}^{n}\right)=t_{j-1 / 2}^{n} \\
\frac{1}{\operatorname{Pr}}\left(1+\frac{4}{3 F}\right)\left(\frac{t_{j}^{n}-t_{j-1}^{n}}{h_{j}}\right)+(1+\alpha)\left(f_{j-1 / 2}^{n} t_{j-1 / 2}^{n}\right)+N b\left(p_{j-1 / 2}^{n} t_{j-1 / 2}^{n}\right)+N t\left(t_{j-1 / 2}^{n}\right)^{2}+\alpha s_{j-1 / 2}^{n-1} u_{j-1 / 2}^{n} \\
-\alpha\left(u_{j-1 / 2}^{n} s_{j-1 / 2}^{n}\right)-\alpha u_{j-1 / 2}^{n-1} s_{j-1 / 2}^{n}-\alpha f_{j-1 / 2}^{n-1} t_{j-1 / 2}^{n}+\alpha t_{j-1 / 2}^{n-1} f_{j-1 / 2}^{n}=\left[R_{2}\right]_{j-1 / 2}^{n-1} \\
\quad h_{j}^{-1}\left(\phi_{j}^{n}-\phi_{j-1}^{n}\right)=g_{j-1 / 2}^{n} \\
\frac{1}{\operatorname{Sc}}\left(\frac{p_{j}^{n}-p_{j-1}^{n}}{h_{j}}\right)+(1+\alpha)\left(f_{j-1 / 2}^{n} p_{j-1 / 2}^{n}\right)+\frac{1}{\operatorname{Sc}}\left(\frac{N t}{N b}\right)\left(\frac{t_{j}^{n}-t_{j-1}^{n}}{h_{j}}\right)+\alpha g_{j-1 / 2}^{n-1} u_{j-1 / 2}^{n} \\
-\alpha\left(u_{j-1 / 2}^{n} g_{j-1 / 2}^{n}\right)-\alpha u_{j-1 / 2}^{n-1} g_{j-1 / 2}^{n}-\alpha f_{j-1 / 2}^{n-1} p_{j-1 / 2}^{n}+\alpha p_{j-1 / 2}^{n-1} f_{j-1 / 2}^{n}=\left[R_{3}\right]_{j-1 / 2}^{n-1}
\end{gathered}
$$

where we have used the abbreviations

$$
\begin{gathered}
\alpha=\frac{\xi^{n-1 / 2}}{k_{n}}, \quad B=\frac{\sin \xi}{\xi} \\
{\left[R_{1}\right]_{j-1 / 2}^{n-1}=-\left[\left(\frac{v_{j}^{n-1}-v_{j-1}^{n-1}}{h_{j}}\right)+(1-\alpha)\left(f_{j-1 / 2}^{n-1} v_{j-1 / 2}^{n-1}\right)-(1+\xi \Lambda-\alpha)\left(u_{j-1 / 2}^{n-1}\right)^{2}\right.} \\
\left.+B\left[s_{j-1 / 2}^{n-1}-N r\left(g_{j-1 / 2}^{n-1}\right)\right]-\frac{1}{\mathrm{Da}} u_{j-1 / 2}^{n-1}\right] \\
{\left[R_{2}\right]_{j-1 / 2}^{n-1}=-\left[\frac{1}{\operatorname{Pr}}\left(1+\frac{4}{3 F}\right)\left(\frac{t_{j}^{n-1}-t_{j-1}^{n-1}}{h_{j}}\right)+(1-\alpha)\left(f_{j-1 / 2}^{n-1} t_{j-1 / 2}^{n-1}\right)+N b\left(p_{j-1 / 2}^{n-1} t_{j-1 / 2}^{n-1}\right)\right.} \\
\left.+N t\left(u_{j-1 / 2}^{n-1}\right)^{2}+\alpha\left(u_{j-1 / 2}^{n-1} s_{j-1 / 2}^{n-1}\right)\right] \\
{\left[R_{3}\right]_{j-1 / 2}^{n-1}=-\left[\frac{1}{\operatorname{Sc}}\left(\frac{p_{j}^{n-1}-p_{j-1}^{n-1}}{h_{j}}\right)+(1-\alpha)\left(f_{j-1 / 2}^{n-1} p_{j-1 / 2}^{n-1}\right)+\alpha\left(u_{j-1 / 2}^{n-1} g_{j-1 / 2}^{n-1}\right)\right.} \\
\left.+\frac{1}{\operatorname{Sc}}\left(\frac{N t}{N b}\right)\left(\frac{t_{j}^{n-1}-t_{j-1}^{n-1}}{h_{j}}\right)\right]
\end{gathered}
$$

The boundary conditions are

$$
f_{0}^{n}=u_{0}^{n}=0, \quad \theta_{0}^{n}=1, \quad \phi_{0}^{n}=1, \quad u_{J}^{n}=0, \quad \theta_{J}^{n}=0, \quad \phi_{J}^{n}=0
$$

\subsection{Phase C: Quasilinearization of Nonlinear Keller Algebraic Equations}

If we assume $f_{j}^{n-1}, u_{j}^{n-1}, v_{j}^{n-1}, s_{j}^{n-1}, t_{j}^{n-1}, g_{j}^{n-1}$, and $p_{j}^{n-1}$ to be known for $0 \leq j \leq J$, then Eqs. (38)-(44) are a system of $7 J+7$ equations for the solution of $7 J+7$ unknowns: $f_{j}^{n}, u_{j}^{n}, v_{j}^{n}, s_{j}^{n}, t_{j}^{n}, g_{j}^{n}$, and $p_{j}^{n}(j=0,1,2, \ldots, J)$. This nonlinear system of algebraic equations is linearized by means of Newton's method as explained in Prasad et al. (2013). 


\subsection{Phase D: Block-Tridiagonal Elimination of Linear Keller Algebraic Equations}

Linear system (38)-(44) can now be solved by the block-elimination method, since they possess a block-tridiagonal structure. Commonly, the block-tridiagonal structure consists of variables or constants, but here, an interesting feature can be observed, namely, that it consists of block matrices. The complete linearized system is formulated as a block matrix system, where each element in the coefficient matrix is a matrix itself. Then, this system is solved using the efficient Keller-box method. The numerical results are affected by the number of mesh points in both directions. After some trials in the $\eta$-direction a larger number of mesh points are selected, whereas in the $\xi$-direction (tangential coordinate) significantly less mesh points are utilized. The $\eta_{\max }$ value has been set at 10 and this defines an adequately large value at which the prescribed boundary conditions are satisfied. The $\xi_{\text {max }}$ value is set at 1.0 for this flow domain. Mesh independence is therefore achieved in the present computations. The computer program of the algorithm is executed in MATLAB running on a PC. The method demonstrates excellent stability, convergence, and consistency, as elaborated by Keller (1978).

\section{RESULTS AND DISCUSSION}

Extensive computations are presented in Figs. 3-21. A much smaller range is necessary for the dimensionless tangential coordinate $(\xi)$. Computations have been conducted (unless otherwise stated) at a general point on the curved surface of the cylinder, i.e., $\xi=\pi / 3$, located at some distance from the lower stagnation point $(\xi \sim 0)$.

Figures 3-5 present typical profiles for velocity, temperature, and concentration for various values of thermophoretic parameter Nt. It can be observed that an increase in thermophoretic parameter Nt leads to a decrease in velocity. The most prominent variation in the profiles arises at intermediate distances from the cylinder surface. However, increasing Nt generates substantial enhancement in both fluid temperature and nanoparticle concentration values. Thermophoresis effectively heats the boundary layer and simultaneously assists particle deposition away from the fluid regime (on to the cylinder surface), thereby accounting for the elevated concentration (nanoparticle) magnitudes in Fig. 5. For $\mathrm{Nt}=0.1$ there is a monotonic decay in nanoparticle concentration from the cylinder wall into the free stream. However, for $\mathrm{Nt}>0.1$, a concentration peak is observed that is progressively displaced further from the cylinder surface, with profiles thereafter decaying to the free stream.

Figures 6-8 depict the influence of Brownian motion parameter $\mathrm{Nb}$ on velocity, temperature, and concentration, where the velocity in the boundary layer increases with rising Brownian motion parameter Nb. Temperature is also slightly elevated. However, the nanoparticle volume fraction profile $(\phi)$ decreases with an increase in Brownian motion parameter Nb. Keblinski et al. (2002) elaborated on various mechanisms that may contribute to enhancement

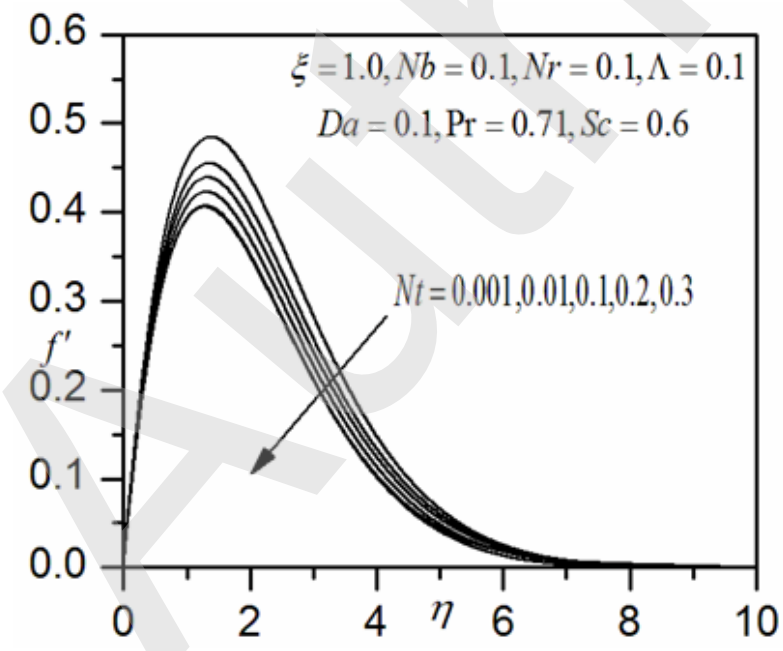

FIG. 3: Influence of $\mathrm{Nt}$ on the velocity profiles

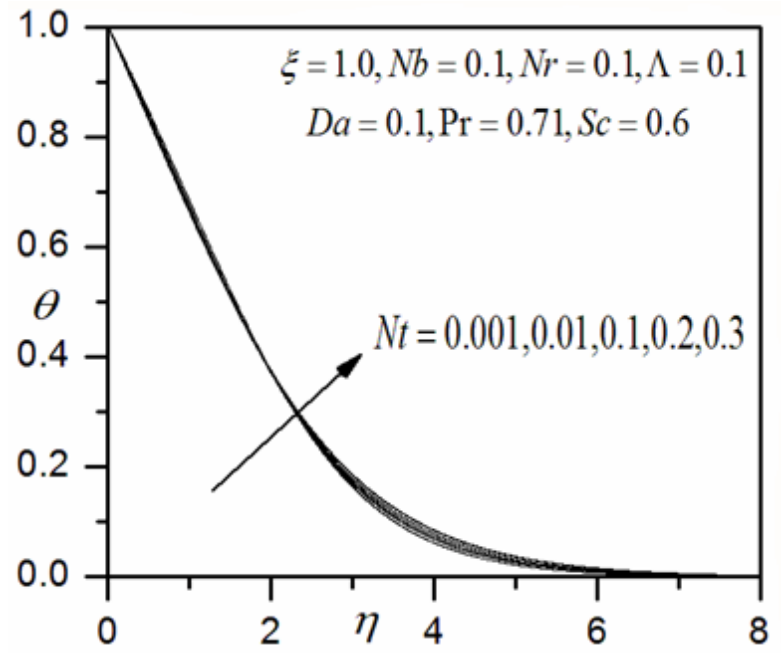

FIG. 4: Influence of Nt on the temperature profiles 


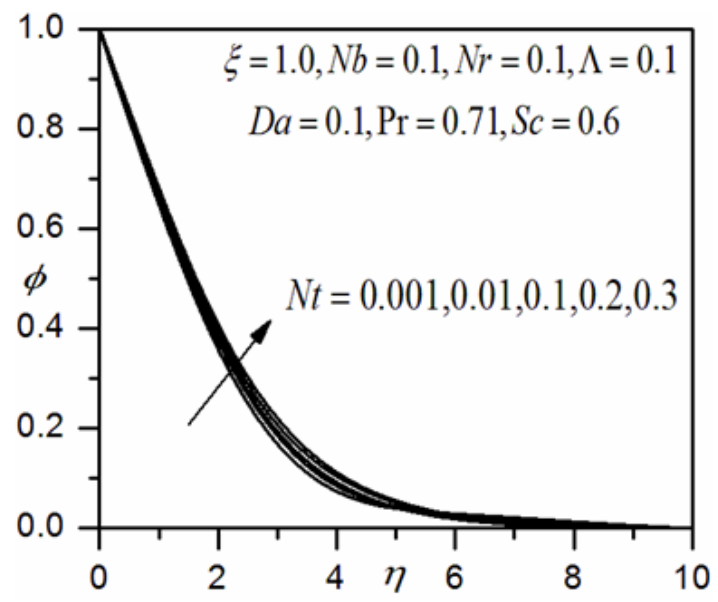

FIG. 5: Influence of $\mathrm{Nt}$ on the concentration profiles

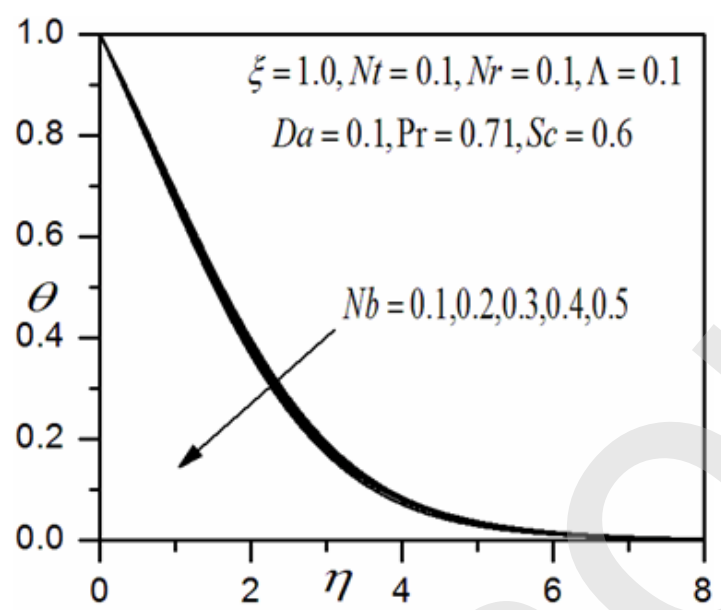

FIG. 7: Influence of $\mathrm{Nb}$ on the temperature profiles

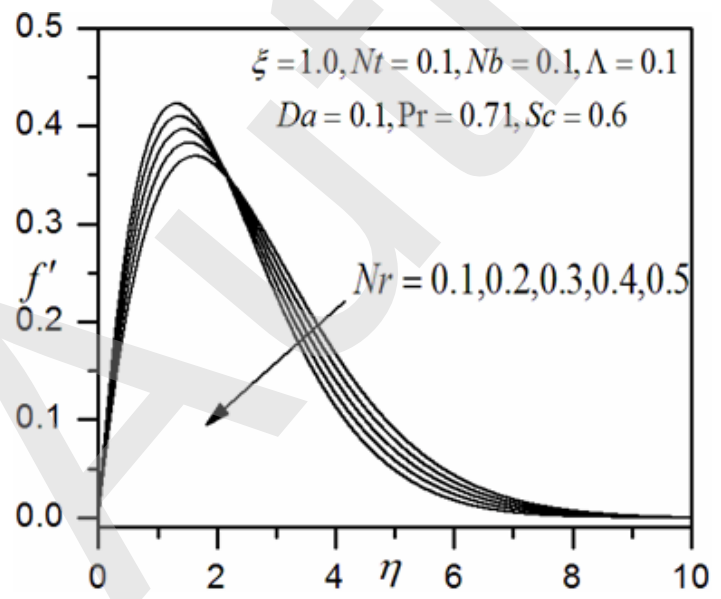

FIG. 9: Influence of $\mathrm{Nr}$ on the velocity profiles

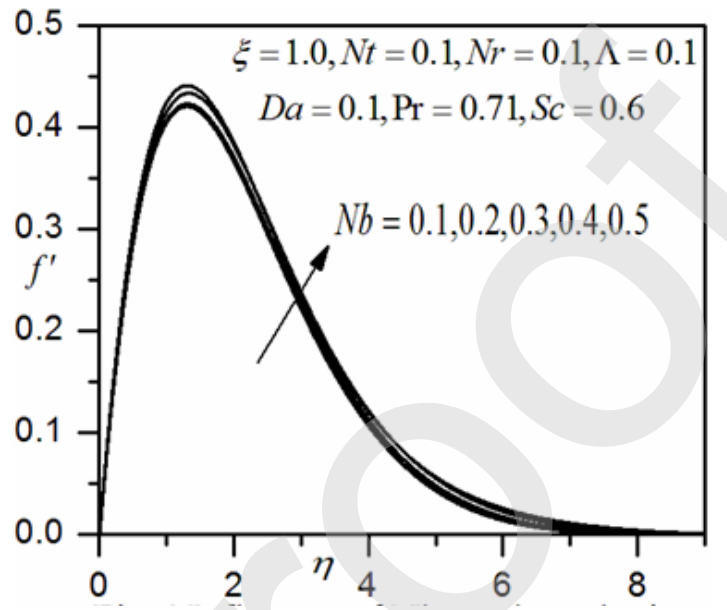

FIG. 6: Influence of $\mathrm{Nb}$ on the velocity profiles

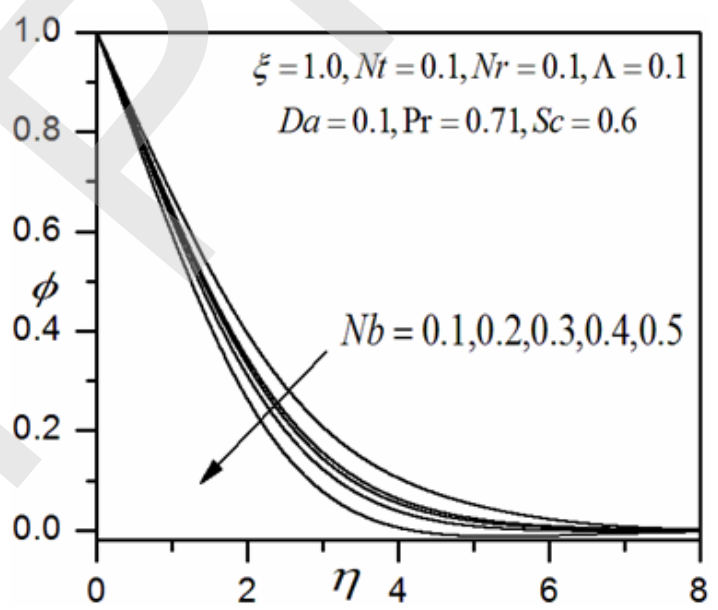

FIG. 8: Influence of $\mathrm{Nb}$ on the concentration profiles

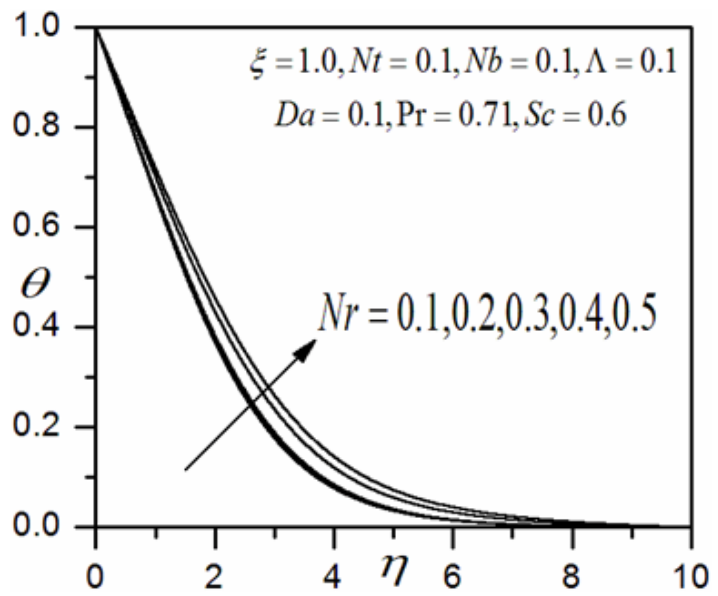

FIG. 10: Influence of $\mathrm{Nr}$ on the temperature profiles 


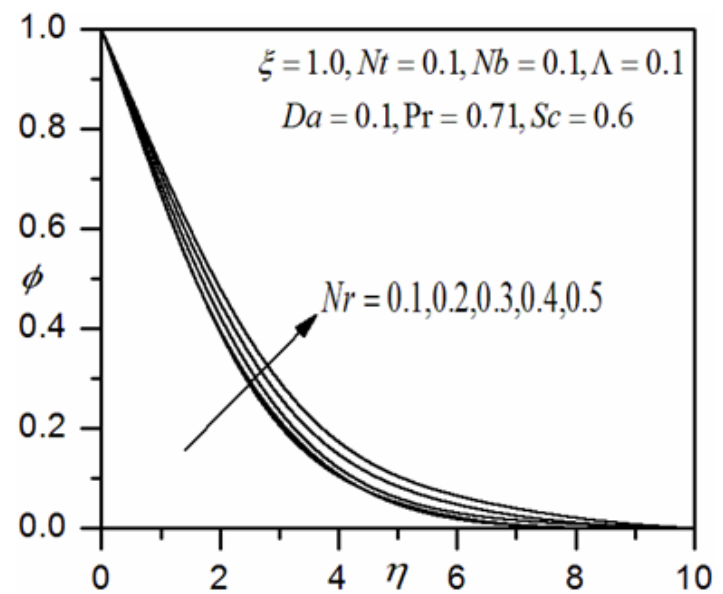

FIG. 11: Influence of $\mathrm{Nr}$ on the concentration profiles

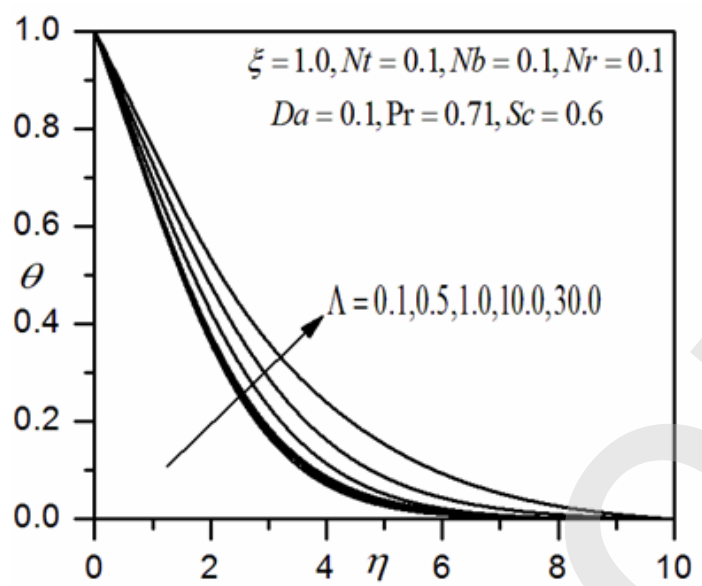

FIG. 13: Influence of $\Lambda$ on the temperature profiles

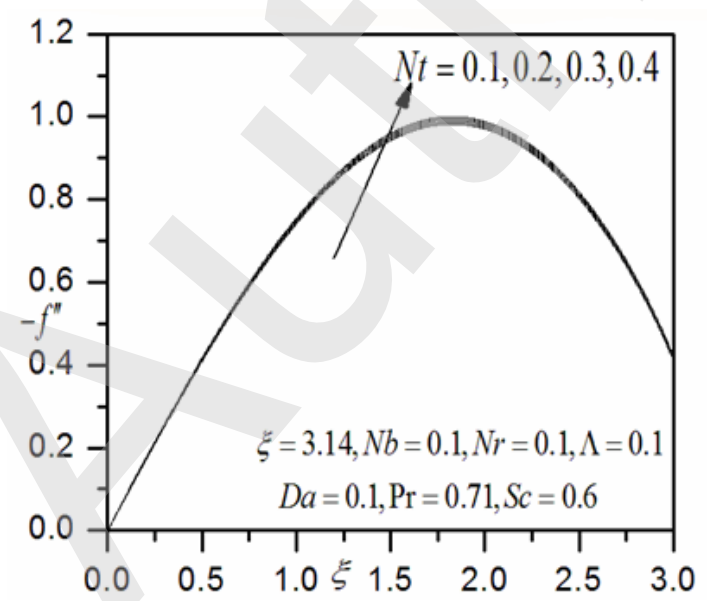

FIG. 15: Effect of $F$ on the skin friction coefficient results

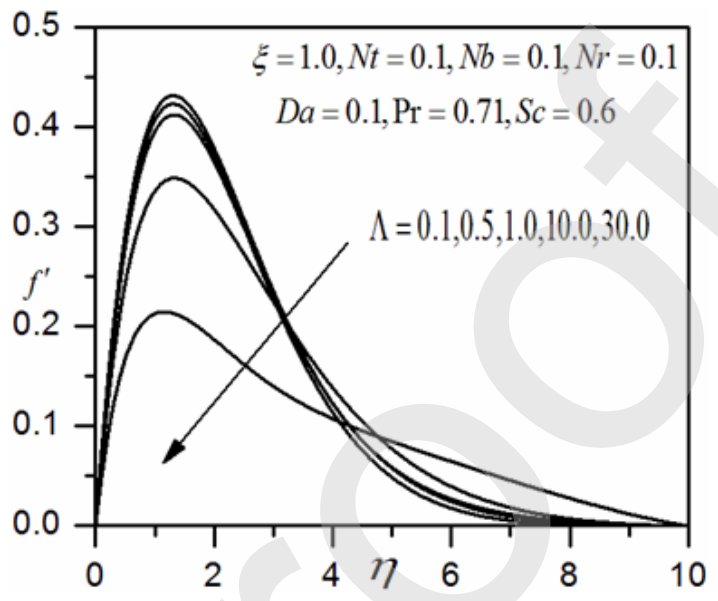

FIG. 12: Influence of $\Lambda$ on the velocity profiles

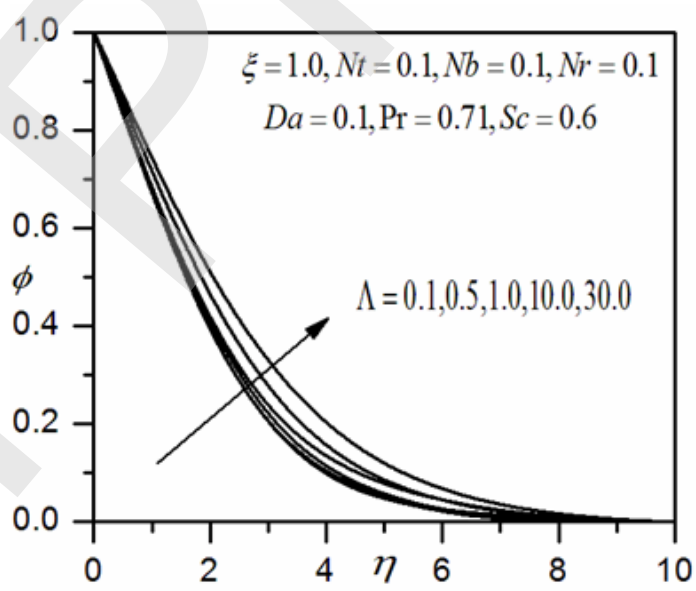

FIG. 14: Influence of $\Lambda$ on the concentration profiles

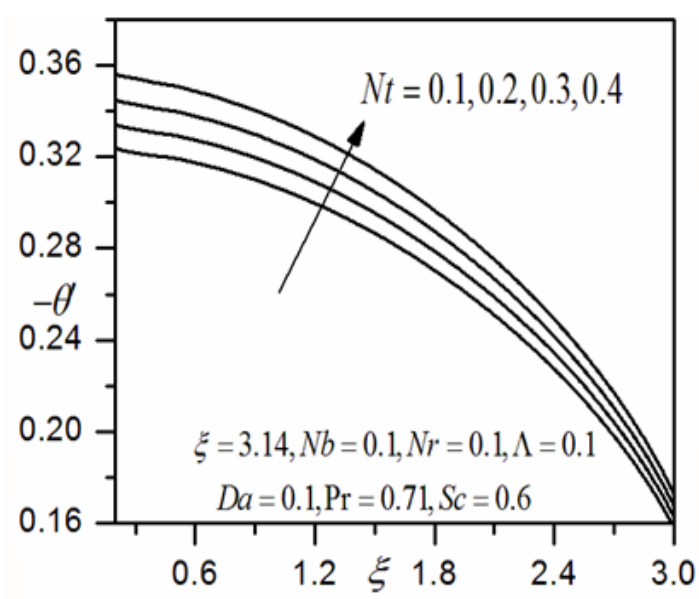

FIG. 16: Effect of $F$ on the local Nusselt number results 


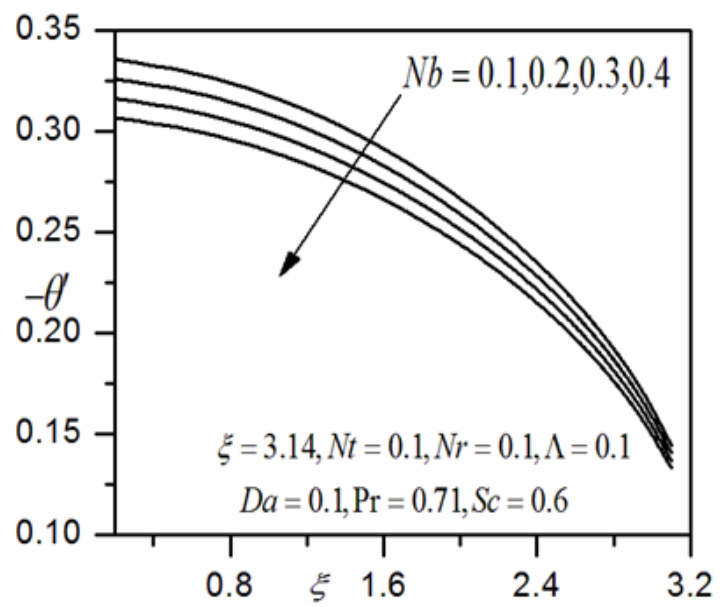

FIG. 17: Effect of $F$ on the local Nusselt number results

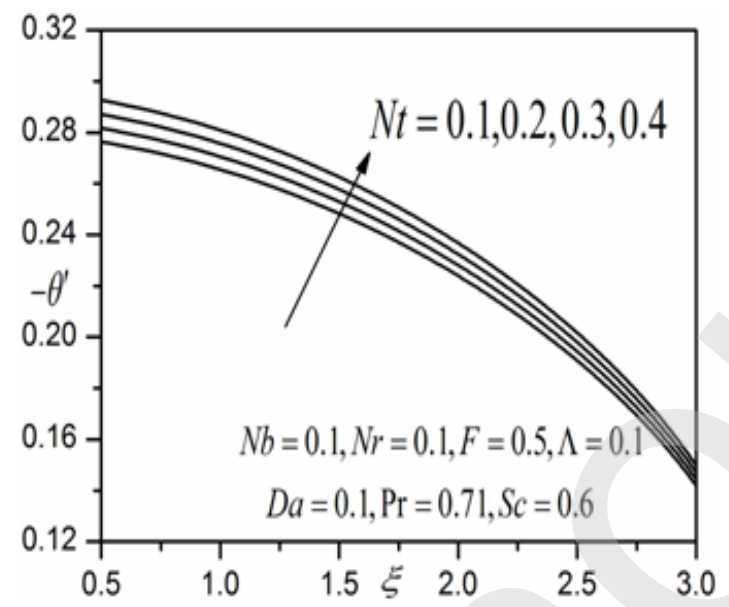

FIG. 19: Effect of Nt on the local Nusselt number results

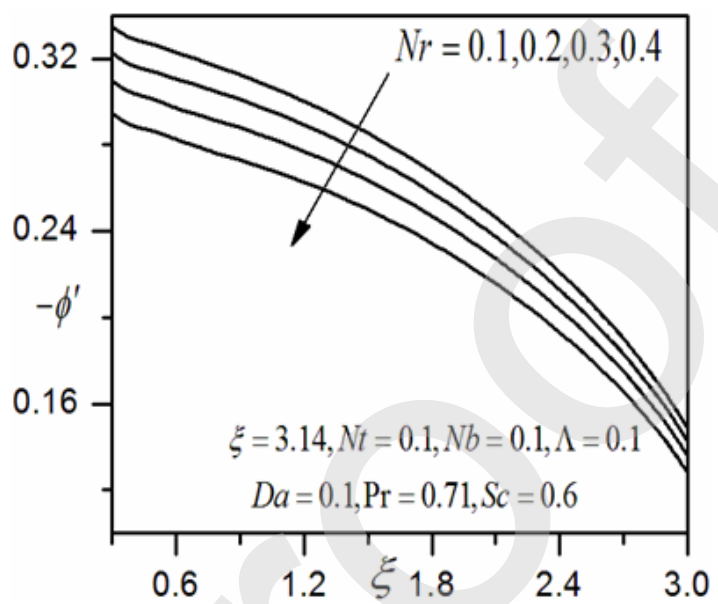

FIG. 18: Effect of Nt on the Sherwood number results

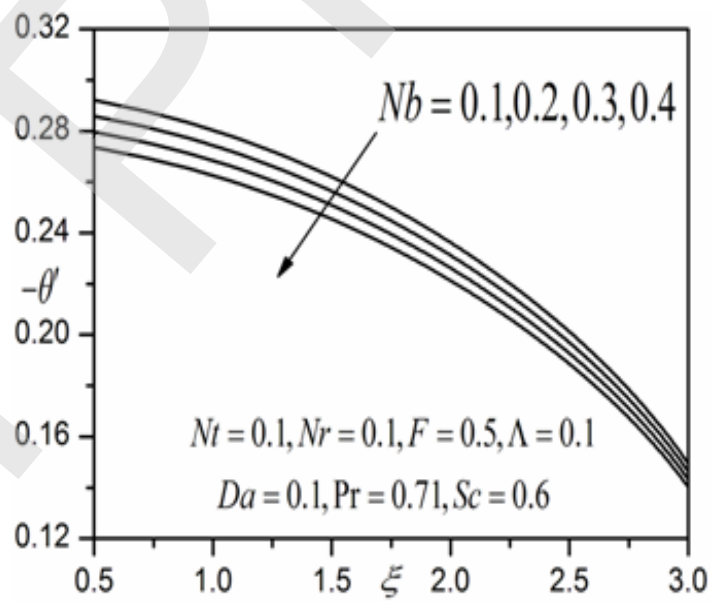

FIG. 20: Effect of $\mathrm{Nb}$ on the local Nusselt number results

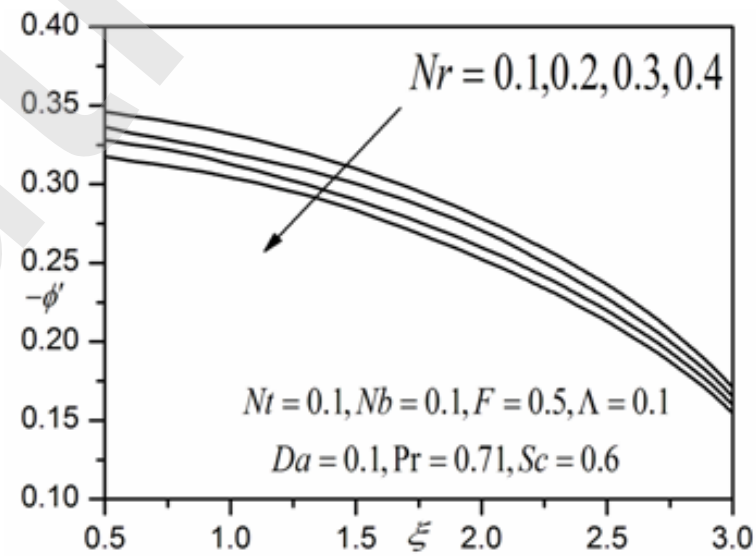

FIG. 21: Effect of $\mathrm{Nr}$ on the Sherwood number results 
in temperatures via augmentation of thermal conductivity with nanoparticles. These include Brownian motion of nanoparticles, ballistic transport of energy carriers within individual nanoparticles and between nanoparticles that are in contact, nanoparticle distribution, and also the interfacial ordering of liquid molecules on the surface of nanoparticles. Brownian motion of nanoparticles may elevate thermal conduction either directly via nanoparticles carrying thermal energy or alternatively via an indirect contribution due to micro-convection of fluid surrounding individual nanoparticles. However, it has been shown that the direct contribution of Brownian motion is less significant since the timescale of the Brownian motion is about two orders of magnitude larger than that for the thermal diffusion of the base liquid. The indirect effect is also argued (Keblinski et al., 2002) to be less significant. Nanoparticles frequently are in the form of agglomerates and/or aggregates. For small particles, Brownian motion is strong and parameter $\mathrm{Nb}$ will have high values; the opposite will apply for large particles. Clearly, Brownian motion does exert an effect on both temperature and concentration profiles, as well as on the momentum field. Flow acceleration is clearly achieved with increasing $\mathrm{Nb}$ values.

Figures 9-11 illustrate the effect of buoyancy ratio parameter $\mathrm{Nr}$ on the velocity $\left(f^{\prime}\right)$, temperature, and concentration distributions through the boundary layer regime

$$
N r=\frac{\left(\rho_{p}-\rho_{f \infty}\right)\left(C_{w}-C_{\infty}\right)}{\rho_{f \infty}\left(1-C_{\infty}\right) \beta\left(T-T_{\infty}\right)}
$$

which represents the relative contribution of the nanoparticle concentration buoyancy force to the thermal buoyancy force. This parameter arises only in momentum boundary layer Eq. (15), in the term ( $\sin \xi / \xi)(\theta-N r \phi)$, and couples the momentum equation to thermal and concentration boundary layer Eqs. (16) and (17), respectively. Velocity is significantly suppressed with increasing Nr. Conversely, both temperature and concentration are markedly enhanced with increasing buoyancy ratio. Therefore, buoyancy forces aid in the diffusion of heat and species (nanoparticles) in the regime, whereas they induce deceleration in the flow regime.

Figures 12-14 show the influence of the Forchheimer inertial parameter $(\Lambda)$ on the flow variables. This parameter is associated with the second-order Forchheimer resistance term, $\xi \Lambda\left(f^{\prime}\right)^{2}$, in momentum Eq. (15). Forchheimer drag is directly proportional to parameter $\Lambda$. An increase in $\Lambda$ markedly decelerates the flow, as illustrated in Fig. 12, for some considerable distance into the boundary layer transverse to the cylinder surface. However, a certain point negligible effects are observed and these may be attributable to the more prominent role of inertial quadratic drag closer to the wall. Kaviany (1992) indicated that Forchheimer effects are associated with higher velocities in porous media transport. However, Forchheimer drag is second order and the increase in this form of drag effectively swamps the momentum development, thereby decelerating the flow, in particular near the cylinder surface. The term non-Darcian does not allude to a different regime of flow but to the amplified effects of Forchheimer drag at higher velocities, as elaborated by Whitaker (1996). With a dramatic increase in $\Lambda$ there is also a slight elevation in temperature (Fig. 13) in the regime. The deceleration in the flow generates a decrease in momentum boundary layer thickness, which aids in energy diffusion and thickening in the thermal boundary layer. The influence on the concentration (nanoparticle species diffusion) field (Fig. 14) is similar to that of the temperature field. However, with the same increment in the Forchheimer parameter, greater disparity in concentration profiles is caused. Concentration is markedly increased, in particular at some distance from the cylinder surface, with an increase in Forchheimer parameter $\Lambda$. As with temperature response, the concentration profiles exhibit a monotonic decay from the cylinder surface to the edge of the boundary layer regime.

Figures 15-17 depict the influence of radiation parameter $F$ on the velocity, temperature, and concentration profiles, where $F=K k^{*} / 4 \sigma^{*} T_{\infty}^{3}$ defines the ratio of the thermal conduction contribution relative to the thermal radiation, and for the radiative heat transfer dominance in the boundary layer regime, $F \rightarrow 0$. For finite values of $F$ there will be a simultaneous presence of thermal conduction and radiative transfer contribution. For $F=1$ both modes will contribute equally. For $F \rightarrow \infty$, in Eq. (16), the term $4 / 3 F \rightarrow 0$ and the energy conservation equation are reduced to the conventional steady conduction-convection equation, i.e.

$$
\frac{1}{\operatorname{Pr}} t^{\prime}+f t+N b(p t)+N t\left(t^{2}\right)=\xi\left(u \frac{\partial s}{\partial \xi}-t \frac{\partial f}{\partial \xi}\right)
$$

Volume 21, Issue 3, 2018 
An increase in $F$ from 0.1 to $0.5,0.1$ to 1.0 , and 3.0 to 5.0 causes a significant increase in velocity with distance into the boundary layer, i.e., it accelerates the flow. Therefore, thermal radiation flux has a retarding effect on the flow regime. This is important in polymeric and other industrial flow processes since it shows that the presence of thermal radiation, while increasing temperature, will affect flow control from the cylinder surface into the boundary layer regime. As expected, temperature values are also significantly enhanced with an increase in $F$ since there is a progressive increase in the thermal radiation contribution accompanying this. Overall, the correct response for temperature is computed with higher radiative flux (lower $F$ values). By increasing the $F$ values, there is a significant decrease in the concentration profiles, as shown in Fig. 17.

Figures 18 and 19 present typical profiles for the dimensionless skin friction coefficient $\left[\xi f^{\prime \prime}(\xi, 0)\right]$, heat transfer rate $\left[\theta^{\prime}(\xi, 0)\right]$, and mass transfer rate $\left[\phi^{\prime}(\xi, 0)\right]$ for various values of thermophoretic parameter Nt. It is evident that the dimensionless skin friction increases very slightly with $\mathrm{Nt}$, whereas the heat transfer rate (local Nusselt number) is considerably increased with an increase in Nt. An increase in thermophoretic parameter Nt leads to a decrease in the mass transfer rate (local Sherwood number). Therefore, thermophoresis exerts a significant effect on both the heat and mass transfer characteristics at the cylinder surface.

Figures 20 and 21 show the influence of Brownian motion parameter $\mathrm{Nb}$ on the dimensionless heat transfer rate $\left[\theta^{\prime}(\xi, 0)\right]$, and buoyancy ratio parameter $\mathrm{Nr}$ on the mass transfer rate $\left[\phi^{\prime}(\xi, 0)\right]$, at the cylinder surface. It is observed that the heat transfer rate is substantially enhanced with increasing $\mathrm{Nb}$. There is also a progressive depletion in the heat transfer rate with increasing the transverse coordinate, i.e., the $\xi$ value. This agrees with the corresponding enhancement in temperature in the boundary layer owing to the nanofluid properties as represented by the Brownian diffusion effect. A decrease in the heat transfer rate at the wall will imply less heat is convected from the fluid regime to the cylinder, thereby heating the boundary layer. The mass transfer rate is enhanced with an increase in the buoyancy ratio since the species buoyancy is accentuated with an increase in the $\mathrm{Nr}$ value, and this aids in boosting the nanoparticle mass transfer rates (the local Sherwood number). In order to verify the accuracy of our present method, we have compared our results with those of Merkin and Pop (1988) and Prasad et al. (2014).

\section{CONCLUSIONS}

A finite-difference numerical solution has been developed for the free convection laminar boundary layer flow from a horizontal cylinder in a nanofluid-saturated non-Darcy porous medium. The computations have shown that the flow is decelerated with an increase in the Forchheimer parameter, whereas the temperature and nanoparticle concentration are both enhanced. An increase in the Brownian motion effect is also found to accelerate the flow and enhance the temperature, whereas the nanoparticle concentration is strongly suppressed. An increase in the thermophoretic parameter induces significant retardation in the flow, whereas it elevates both temperatures and nanoparticle concentrations. Furthermore, an increase in the buoyancy parameter decelerates the flow, whereas it elevates both temperatures and nanoparticle concentrations in the boundary layer regime. The present study has been confined to steady-state flow and ignored swirling effects in nanofluids.

\section{ACKNOWLEDGMENTS}

The authors appreciate the constructive comments of the reviewers, which led to definite improvement in the paper. The corresponding author, Dr. A. Subba Rao, thanks the University Grants Commission (SERO), Hyderabad, India, for financial support under a Minor Research Project grant [MRP-4613/14(2014)], as well as the management of the Madanapalle Institute of Technology and Science, Madanapalle, India.

\section{REFERENCES}

Bear, J., Dynamics of Fluids in Porous Media, New York, NY: Dover, 1988.

Bég, O.A., Numerical Methods for Multi-Physical Magneto Hydrodynamics, chap. 1, in New Developments in Hydrodynamics Research, M.J. Ibragimov and M.A. Anisimov, Eds., New York, NY: Nova Science, pp. 1-10, 2012. 
Bég, O.A., Bakier, A.Y., and Prasad, V.R., Numerical Study of Free Convection Magnetohydrodynamic Heat and Mass Transfer from a Stretching Surface to a Saturated Porous Medium with Soret and Dufour Effects, Comput. Mater. Sci., vol. 46, pp. 57-65, 2009.

Bég, O.A., Bég, T.A., Takhar, H.S., and Raptis, A., Mathematical and Numerical Modeling of Non-Newtonian ThermoHydrodynamic Flow in Non-Darcy Porous Media, Int. J. Fluid Mech. Res., vol. 31, pp. 1-12, 2004.

Bég, O.A., Bhargava, R., Rawat, S., Takhar, H.S., and Bég, T.A., A Study of Buoyancy Driven Dissipative Micro Polar Free Convection Heat and Mass Transfer in a Darcian Porous Medium with Chemical Reaction, Nonlinear Analysis, Model. Control J., vol. 12, pp. 157-180, 2007.

Bég, O.A., Makinde, O.D., Zueco, J., and Ghosh, S.K., Hydromagnetic Viscous Flow in a Rotating Annular High-Porosity Medium with Nonlinear Forchheimer Drag Effects: Numerical Study, World J. Modell. Simul., vol. 8, pp. 83-95, 2012.

Bég, O.A., Takhar, H.S., Bharagava, R., Rawat, S., and Prasad, V.R., Numerical Study of Heat Transfer of a Third Grade Viscoelastic Fluid in Non-Darcian Porous Media with Thermo-Physical Effects, Phys. Scr., vol. 77, pp. 1-11, 2008.

Bejan, A. and Khair, K.R., Heat and Mass Transfer by Natural Convection in a Porous Medium, Int. J. Heat Mass Transf., vol. 28, pp. 909-918, 1985.

Buongiorno, J., Convective Transport in Nanofluids, ASME J. Heat Transf., vol. 128, pp. 240-250, 2006.

Buongiorno, J. and Hu, W., Nanofluid Coolants for Advanced Nuclear Power Plants, in Proc. of International Congress on Advances in Nuclear Power Plants (ICAPP'05), Seoul, Korea, ICAPP-5705, 2005.

Cai, J. and Yu, B., A Discussion of the Effect of Tortuosity on the Capillary Imbibition in Porous Media, Transp. Porous Media, vol. 89, pp. 251-263, 2011.

Chamkha, A.J, Takhar, H.S., and Bég, O.A., Radiative Free Convective Non-Newtonian Fluid Flow past a Wedge Embedded in a Porous Medium, Int. J. Fluid Mech. Res., vol. 31, pp. 101-115, 2004.

Das, S.K., Choi, S., Yu, W., and Pradeep, T., Nanofluids: Science and Technology, Hoboken, NY: Wiley InterScience, 2007.

Dorfman, K.D. and Brenner, H, Generalized Taylor-Aris Dispersion In Discrete Spatially Periodic Networks: Microfluidic Applications, Phys. Rev. E, vol. 65, pp. 20-37, 2002.

Hossain, M.A. and Pop, I., Studied Radiation Effects on Free Convection over a Flat Plate Embedded in a Porous Medium with High-Porosity, Int. J. Therm. Sci., vol. 40, pp. 289-295, 2001.

Kaviany, M., Principles of Heat Transfer in Porous Media, New York, NY: McGraw-Hill, 1992.

Keblinski, P., Phillpot, S.R., Choi, S.U.S., and Eastman, J.A., Mechanisms of Heat Flow in Suspensions of Nanosized Particles (Nanofluids), Int. J. Heat Mass Transf., vol. 45, pp. 855-863, 2002.

Keller, H.B., Numerical Methods in Boundary-Layer Theory, Annu. Rev. Fluid Mech., vol. 10, pp. 417-433, 1978.

Lai, F.C., and Kulacki, F.A., Coupled Heat and Mass Transfer by Natural Convection from Vertical Surface in Porous Medium, Int. J. Heat Mass Transf., vol. 34, pp. 1189-1194, 1991.

Merkin, J.H., and Pop, I., A Note on the Free Convection Boundary Layer on a Horizontal Circular Cylinder with Constant Heat Flux, Warme Stoffubertr., vol. 22, pp. 79-81, 1988.

Modest, M.F., Radiation Heat Transf., New York, NY: McGraw-Hill, 1993.

Murthy, P.V.S.N. and Singh, P., Heat and Mass Transfer by Natural Convection in a Non-Darcian Porous Medium, Acta Mech., vol. 138, pp. 243-254, 1999.

Nagaraju, P., Chamkha, A.J., Takhar, H.S., and Chandrasekhara, B.C., Simultaneous Radiative and Convective Heat Transfer in a Variable Porosity Medium, Heat Mass Transfer J., vol. 37, pp. 243-250, 2001.

Nield, D.A. and Kuznetsov, A.V., Thermal Instability in a Porous Medium Layer Saturated by a Nanofluid, Int. J. Heat Mass Transf., vol. 52, pp. 5796-5801, 2009.

Pomès, V., Fernández, A., and Houi, D, Characteristic Time Determination for Transport Phenomena during the Electro Kinetic Treatment of a Porous Medium, Chem. Eng. J., vol. 87, pp. 251-260, 2002.

Prasad, V.R., Gaffar, S.A., and Bég, O.A., Heat and Mass Transfer of a Nanofluid from Horizontal Cylinder to Micropolar Fluid, J. Thermophys. Heat Transf., vol. 29, no. 1, pp. 127-139, 2015.

Prasad, V.R., Rao, S.A., and Bég, O.A., Computational Analysis of Viscous Dissipation and Joule Heating Effects on Non-Darcy MHD Natural Convection Flow from a Horizontal Cylinder in Porous Media with Internal Heat Generation, Theor. Appl. Mech., vol. 41, pp. 37-70, 2014.

Volume 21, Issue 3, 2018 
Prasad, V.R., Rao, A.S., Reddy, N.B., and Bég, O.A., Modelling Laminar Transport in a Casson Fluid from an Isothermal Sphere with Partial Slip in a Non-Darcy Porous Medium, Theor. Appl. Mech., vol. 40, pp. 469-510, 2013.

Rashidi, M.M., Freidoonimehr, N., Hosseini, A., Bég. O.A., and Hung, T.K., Homotopy Simulation of Nanofluid Dynamics from a Non-Linearly Stretching Isothermal Permeable Sheet with Transpiration, Meccanica, vol. 49, pp. 469-482, $2014 \mathrm{a}$.

Rashidi, M.M., Hosseini, A., Pop, I., Kumar, S., and Freidoonimehr, N., Comparative Numerical Study of Single and Two-Phase Models of Nanofluid Heat Transfer in Wavy Channel, Appl. Math. Mech., vol. 35, pp. 831-848, 2014 b.

Rashidi, M.M, Momoniat, E., Ferdows, M., and Basiriparsa, A., Lie Group Solution for Free Convective Flow of a Nanofluid past a Chemically Reacting Horizontal Plate in a Porous Media, Math. Problems Eng., vol. 2014, Article ID 239082, 2014c.

Rashidi, M.M., Vishnu Ganesh, N., Abdul Hakeem, A.K., and Ganga, B., Buoyancy Effect on MHD Flow of Nanofluid over a Stretching Sheet in the Presence of Thermal Radiation, J. Mol. Liq., vol. 198, pp. 234-238, 2014 d.

Raptis, P. and Perdikis, C., Unsteady Flow through a Highly Porous Medium in the Presence of Radiation, Transp. Porous Media, vol. 57, pp. 171-179, 2004.

Subba Rao, A., Prasad, V.R., Harshavalli, K., and Beg, O.A., Thermal Radiation Effects on Non-Newtonian Fluid in a Variable Porosity Regime with Partial Slip, J. Porous Media, vol. 19, no. 4, pp. 313-329, 2016 a.

Subba Rao, A., Prasad, V.R., Nallagundla, N., Bhaskar Reddy, N., and Bég, O.A., Non-Similar Computational Solution for Boundary Layer Flows of Non-Newtonian Fluid from an Inclined Plate with Thermal Slip, J. Appl. Fluid Mech., vol. 9, pp. 795-807, 2016b.

Takhar, H.S., Bég, O.A., Chamkha, A.J., Filip, D., and Pop, I., Mixed Radiation-Convection Boundary Layer Flow of an Optically Dense Fluid along a Vertical Flat Plate in a Non-Darcy Porous Medium, Int. J. Appl. Mech. Eng., vol. 8, pp. 483-496, 2003.

Takhar, H.S., Bég, O.A., and Kumari, M., Computational Analysis of Coupled Radiation- Convection Dissipative Non-Gray Gas Flow in a Non-Darcy Porous Medium using the Keller-Box Implicit Difference Scheme, Int. J. Energy Res., vol. 22, pp. 141159,1998

Takhar, H.S., Chamkha, A.J., and Nath, G., Natural Convection on a Vertical Cylinder Embedded in a Thermally Stratified HighPorosity Medium, Int. J. Therm. Sci., vol. 41, pp. 83-93, 2002.

Tham, L., Nazar, R., and Pop, I., Mixed Convection Boundary Layer Flow from a Horizontal Circular Cylinder in a Nanofluid, Int. J. Numer. Methods Heat Fluid Flow, vol. 22, pp. 576-606, 2012

Turner, I.W., Puiggali, J.R., and Jomaa, W., A Numerical Investigation of Combined Microwave and Convective Drying of a Hygroscopic Porous Material: A Study Based on Pine Wood, Chem. Eng. Res. Des., vol. 76, pp. 193-209, 1998.

Tzou, D.Y, Thermal Instability of Nanofluids in Natural Convection, Int. J. Heat Mass Transf., vol. 51, pp. $2967-2979,2008$.

Whitaker, S., The Forchheimer Equation: A Theoretical Development, Transp. Porous Media, vol. 25, pp. 27-62, 1996.

Xiao, B., Yang, Y., and Chen, L., Developing a Novel Form of Thermal Conductivity of Nanofluids with Brownian Motion Effect by Means of Fractal Geometry, Powder Technol., vol. 239, pp. 409-414, 2013.

Yacob, N.A., Ishak, A., and Pop, I., Falkner-Skan Problem for a Static or Moving Wedge in Nanofluids, Int. J. Therm. Sci., vol. 2, pp. 133-139, 2011.

Yu, D., Routbort, J.L., and Choi, S., Review and Comparison of Nanofluid Thermal Conductivity and Heat Transfer Enhancements, Heat Transf. Eng., vol. 29, pp. 432-460, 2008.

Zueco, J. and Bég, O.A., Network Simulation Solutions for Laminar Radiating Dissipative Magneto-Gas Dynamic Heat Transfer over a Wedge in Non-Darcian Porous Regime, Math. Comput. Modell., vol. 50, pp. 439-452, 2009. 